\title{
Impact of Renewable Energy Sources on Relay Protection Operation
}

\author{
M.V. Andreev ${ }^{1, a)}$, M.A. Denisov ${ }^{1, b)}$ and A.S. Gusev ${ }^{1, c)}$ \\ ${ }^{1}$ National Research Tomsk Polytechnic University, 634050 Tomsk, Russia. \\ a)Corresponding author: andreevmv@tpu.ru \\ b) garret-9696@mail.ru \\ c)gusev_as@tpu.ru
}

\begin{abstract}
The current trend of any electric power system is the integration of renewable energy sources (RES). Mostly these are solar and wind power plants. The penetration of renewable energy leads to significant changes in the operating mode of the power system and, accordingly, affects the functioning of the relay protection and automation devices. In particular, the use of renewable energy can lead to a decrease in short-circuit currents and the insensitivity of the protection to this fault. The paper demonstrates the results of a study that confirmed this. In the paper analyzed the existing approaches to setting up relay protection and automation. Based on this analysis, conclusions are made.
\end{abstract}

\section{INTRODUCTION}

All experts agreed about necessity and inevitability of renewable energy sources (RES) integration. This process is already begun and continues at different speeds in different countries. The generated power of sources working on wind and solar energy has increased by more than $30 \%$ over the past 15 years.

One of the main reasons hindering the integration of RES is its impact on the operating modes of electrical power systems (EPS), which in turn has a significant effect at the functioning of relay protection (RP) devices. Distribution networks, as a rule, are protected by overcurrent protection (OCP). This type of protection, however, is applicable only to radial networks with one-sided power supply. In the case of networks of more complex configuration, directional OCP (DOCP) is used, since the only current value control is not allow provide selectivity of RPs operation. The use of DOCP is also limited: they are not installing in networks with several power sources. That is why they not used in EPS with RES. The value of short circuit current (SCC) and its direction depends on the type, quantity and location of RES in the network [1]. The main problems of ensuring the correct functioning of protection during the integration of renewable energy sources are: 1) insensitivity of RP; 2) RP' false tripping; 3) EPS partitioning problems; 4) non-selectivity of RP actions; 5) problems with auto reclosers.

\section{RESEARCH RESULTS}

The above features of EPS with RES are confirmed by the following studies for the 14-bus IEEE scheme (Fig.1).

Case 1: 1) the methodology for carrying out experiments was adopted in accordance with [2]; 2) installation place for a wind power plant (WPP) is bus $14(110 \mathrm{kV})$.

Experiment No. 1 - Phase-to-phase short circuit (AB) at the bus 13 when the WPP generated power changes from 0 to $30 \mathrm{MW}$ at the bus 14. The nature of the SCC changes on the line L-17 is interesting (Fig. $2 \mathrm{a}$ ) - since the wind farm power increase, the magnitude of SCC flowing through the line L-17 is decrease in case of the phase-tophase short circuit at the bus 13. Thus, the RP installed at the beginning of the L-17 (considering the bus 9 as the beginning of power transmission line and the bus 14 as an end) for a certain power of the WPP will not be effective as a back-up protection of the line L-20 (the SCC value will be less than the protection pick up current).

International Youth Scientific Conference "Heat and Mass Transfer in the Thermal Control System of Technical and Technological Energy Equipment" (HMTTSC 2019) AIP Conf. Proc. 2135, 020001-1-020001-3; https://doi.org/10.1063/1.5120638 Published by AIP Publishing. 978-0-7354-1878-3/\$30.00 
Experiment No. 2 - Three-phase short-circuit at the bus 13 when the WPP generated power changes from 0 to 30 MW at the bus 14. In case of three-phases short-circuit at the bus 13 and increment of the WPP power, the SCC in the line L-17 increases (Fig. 2 b).

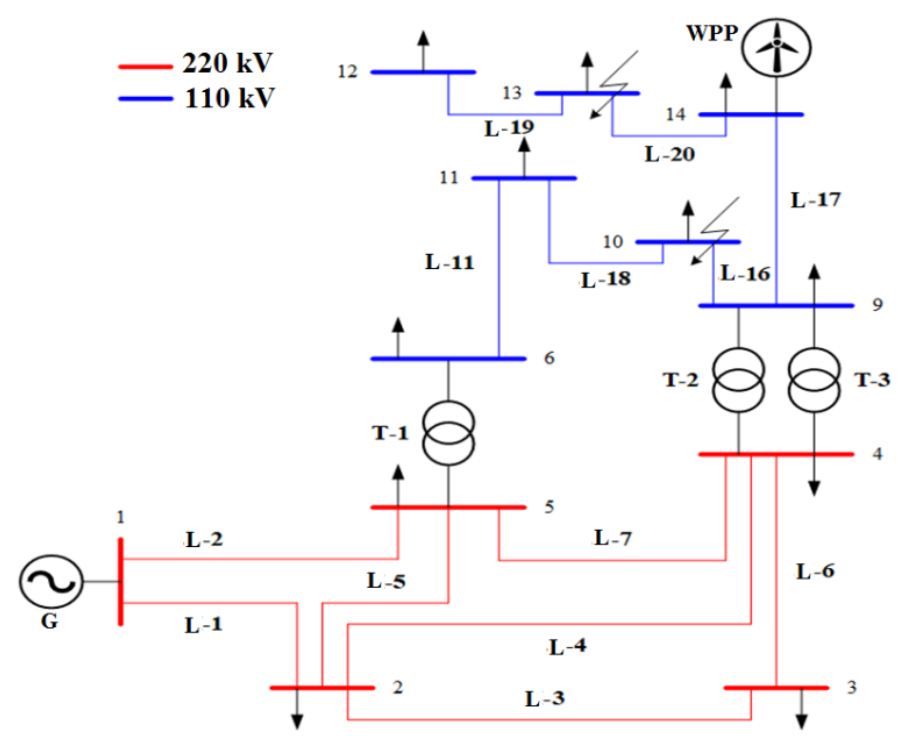

FIGURE 1. Test scheme of EES for research - IEEE 14-Bus System

Experiment No. 3 - The WPP with generated power $10 \mathrm{MW}$ installed at the bus 13 , test disturbance is a phaseto-phase short circuit $(\mathrm{AB})$. In this case the SCC flowing through the line L-17 firstly decreases (for $\mathrm{x}<0.5 \mathrm{pu}$ ), but then its increase (at $x>0.5 \mathrm{pu}$ ). Thus, the relay protection installed at the beginning of the L-17 for a certain location of the WPP will not operate effectively as a back-up protection of the line L-20 (the SCC will be less than protection pick up current).

Case 2: The WPP with generated power $30 \mathrm{MW}$ installed at the bus 14, test disturbance is a three-phase short circuit at the bus 10 (Fig. 1).

For this case are following two problems were investigated: 1) problem 1 - changing direction of current flow in both steady-state and emergency (short circuit) modes; 2) problem 2 - increasing magnitude of the SCC flowing through power lines after external short-circuits, and therefore non-selective tripping of relay protection is possible (disconnection of power lines that are not damaged $\rightarrow$ possible cascading disconnection of other power lines).

Experiment No. 1 - without WPP: in normal mode, the current through the line L-17 flows from the bus 9 towards the bus 14 , when a short circuit occur current in the line is decreasing, since all currents flows to the place of short circuit.

Experiment No. 2 - WPP is installed with generated power $30 \mathrm{MW}$ at the bus 14 . In steady-state mode, the current in the line L-17 already flows from the bus 14 to the bus 9 (the current flow direction changes), and after short circuit the current' magnitude increases due to the appearance of additional feed for SCC from the wind farm.

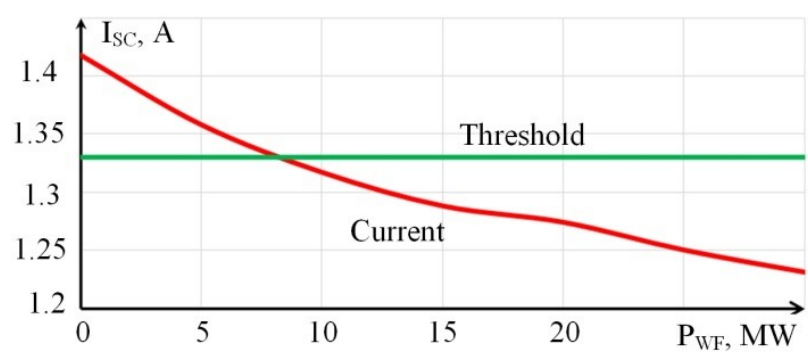

(a)

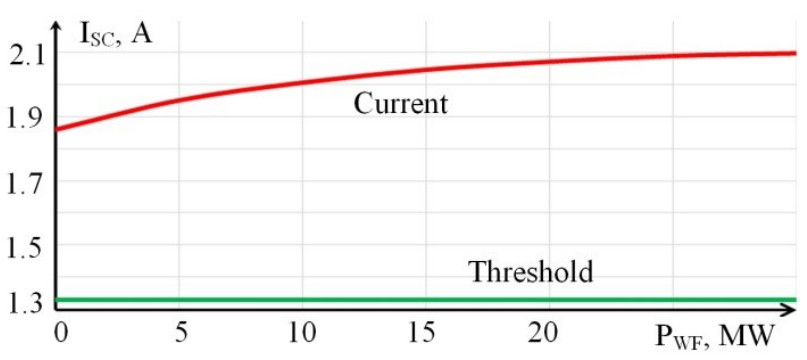

(b)

FIGURE 2. The magnitude of the SCC flowing through the line L-17, with a phase-to-phase short circuit (AB) at the bus 13: curve - short-circuit current; horizontal line - RP threshold at the beginning of the line L-17. 


\section{CONCLUSION}

The carried out experiments shown, that traditional principles of power facilities protection are not suitable for distribution networks with renewable energy sources. The following discusses existing approaches to protecting such networks.

1) Distance protection of distribution systems [3]. Distance protections (DP) are the widest spread in EPS. In case of bulk penetration of renewable energy sources, it is extremely difficult to take into account the transient impedance, so the protection zone on the DP characteristic can be both excessive and insufficient, which can cause wrong actions.

2) As a solution of the problem of RP setting up, ensuring its correct functioning in modern EPS, algorithms are proposed for determining the capacity and locations of the RES installation in such a way that it does not affect the RP settings and, accordingly, their operation. This approach eliminates the need for a significant update of existing methods for the settings calculation, however, hinders the integration of RES.

3) In [4], an approach to constructing a RP is proposed, which eliminates the need for settings calculation at whole - a method of states estimation (setting-less). Based on analysis, it seems that this method requires an unnecessarily large number of measurable parameters, as well as verification according to many physical laws. This complicates the real scheme of protection connection to the object in the EPS. It is also not clear how this model takes into account the errors of the instrument transformers, especially their non-linear change due to saturation, as well as the internal elements of the RP scheme: auxiliary converters and filters. In addition, it is necessary to note that practical experience with setting-less protection is absent in the world, so it's hard to assess its reliability. The application of this approach will require radical changes in the construction of protection systems, as well as the replacement of those already installed. The existing algorithms proved their efficiency and high reliability.

Summarizing the above, it is possible to state with confidence the need to develop new methods and tools for PR setting up for EPS with RES, since the existing approaches either limit the integration of new equipment and not flexible enough. The other aspects of the mentioned problem discussed by authors in [5-6].

\section{ACKNOWLEDGMENTS}

The reported study was funded by Ministry of Science and Higher Education of Russian Federation, according to the Agreement No. 075-02-2018-271 "Investigation of the influence of the spectrum of processes in electric power systems with a significant share of distributed generation and renewable energy sources on the functioning of relay protection devices and the development of a methodology for its adequate setting up".

\section{REFERENCES}

1. V. Telukunta, J. Pradhan, A. Agrawal, M. Singh and S. G. Srivani, JPES 3, 365-379 (2017).

2. H. Zhan, C. Wang, Y. Wang, X. Yang, X. Zhang, C. Wu and Y. Chen, IEEE Transactions on Smart Grid 7, 55-65 (2016).

3. A. Sinclair, D. Finney, D. Martin and P. Sharma, IEEE Transactions on Industry Applications 50, $2186-2196$ (2014).

4. A. Sinclair, D. Finney, D. Martin and P. Sharma, IEEE Transactions on Power Delivery 32, 320-330 (2017).

5. R.A. Ufa, A.S. Vasiliev, A.S. Gusev, and A.A. Suvorov, "Development of hybrid model of STATCOM," in International Forum on Strategic Technology IFOST-2016, Conference Proceedings 596, edited by F. Byk (Novosibirsk State Technical University, Novosibirsk, 2016), pp. 113-117.

6. N.Y. Ruban, A.O. Sulaymanov, R.A. Ufa and I.A. Razzhivin, MATEC Web Conf. 91, 1-4 (2017). 